\title{
BMJ Open Evidence synthesis, practice guidelines and real-world prescriptions of new generation antidepressants in the treatment of depression: a protocol for cumulative network meta-analyses and meta-epidemiological study
}

\author{
Yan Luo, ${ }^{1}$ Anna Chaimani, ${ }^{2,3,4}$ Yuki Kataoka, ${ }^{5}$ Edoardo Giuseppe Ostinelli, ${ }^{6}$ \\ Yusuke Ogawa, ${ }^{1}$ Andrea Cipriani, ${ }^{7}$ Georgia Salanti, ${ }^{8}$ Toshi A Furukawa ${ }^{1}$
}

To cite: Luo Y, Chaimani A, Kataoka Y, et al. Evidence synthesis, practice guidelines and real-world prescriptions of new generation antidepressants in the treatment of depression: a protocol for cumulative network meta-analyses and meta-epidemiological study. BMJ Open 2018;8:e23222. doi:10.1136/ bmjopen-2018-023222

- Prepublication history for this paper is available online. To view these files, please visit the journal online (http://dx.doi org/10.1136/bmjopen-2018023222).

Received 27 March 2018 Revised 18 August 2018 Accepted 16 October 2018

Check for updates

(c) Author(s) (or their employer(s)) 2018. Re-use permitted under CC BY-NC. No commercial re-use. See rights and permissions. Published by BMJ.

For numbered affiliations see end of article.

Correspondence to Professor Toshi A Furukawa; furukawa@kuhp.kyoto-u.ac.jp

\section{ABSTRACT}

Introduction Depressive disorders are the most common, burdensome and costly mental disorders. Their treatments have developed through the past decades and we now have more than a dozen new generation antidepressants, while a series of guidelines have been published to provide recommendations over the years. However, there still may exist important gaps in this evidence synthesis and implementation process. Systematic reviews may not have been conducted in the most unbiased, informative and timely manners; guidelines may not have reflected the most up-to-date evidence; clinicians may not have changed their clinical decision-makings in accordance with the relevant evidence. The aim of this study is to examine the gaps between the ideally synthesised evidence, guideline recommendations and real-world clinical practices in the prescription of new generation antidepressants for major depression through the past three decades.

Methods and analysis We will conduct cumulative network meta-analyses (cNMAs) based on the comprehensive systematic review which has identified published and unpublished head-to-head randomised controlled trials comparing the following antidepressants in the acute phase treatment of major depression: agomelatine, amitriptyline, bupropion, citalopram, clomipramine, desvenlafaxine, duloxetine, escitalopram, fluoxetine, fluvoxamine, levomilnacipran, milnacipran, mirtazapine, nefazodone, paroxetine, reboxetine, sertraline, trazodone, venlafaxine, vilazodone and vortioxetine. The primary outcomes will be the proportions of patients who responded (efficacy) and who withdrew from treatment for any reasons (acceptability). We will conduct a random effects cNMA to synthesise evidence and obtain a comprehensive ranking of all new generation antidepressants based on their surface under the cumulative ranking curves. We will identify series of international clinical practice guidelines for the treatment of major depression of adults and summarise their recommendations. We will estimate real-world prescription patterns of antidepressants in the nationally representative

\section{Strengths and limitations of this study}

- This is one of the first studies to directly compare evidence derived from network meta-analyses, recommendations from guidelines and real-world clinical practices.

- This study will show where the gaps may lie and how big they are, which can inform future efforts to bridge the gaps from scientific evidence to real-world clinical practices.

- The cumulative network meta-analysis used in this study may evolve into prospectively designed sequential network meta-analysis which can be used as living accumulation of evidence and contribute to living guidelines.

- A major limitation of our study is that the real-world practices in nationally representative samples are available for USA only.

samples in USA in the Medical Expenditure Panel Survey. We will compare and evaluate the gaps between the rankings according to cNMAs conducted at 5-year intervals between 1990 and 2015, recommendations in guidelines published in the ensuing 5 years and actual practices thereafter.

Ethics and dissemination This review does not require ethical approval. We will disseminate our findings through publications in peer-reviewed journals and presentations at conferences.

Trial registration number UMIN000031898.

\section{INTRODUCTION}

Evidence-based medicine (EBM) is widely regarded as the guiding principle of today's medical practices, for it can help integrate patients' values, physicians' experiences and scientific evidence derived from large-scale research to inform clinical decision-making. ${ }^{1}$ 
To promote EBM, systematic reviews (SRs) including meta-analyses (MAs) should be conducted to produce a comprehensive summary of all relevant studies, clinical practice guidelines (CPGs) will be proposed based on such evidence synthesis, and physicians must update their shared decision-making processes in accordance with such guidelines. ${ }^{2}$

Depressive disorders are the most common mental disorders, making them the second leading cause of disability accounting for $8.2 \%$ of all years lived with disability in $2010 .^{3-5}$ Reflecting the high prevalence of major depressive disorder (MDD) among people of working ages and the resulting dysfunctions, their economic burden was estimated at US\$210.5 billion in US alone in $2010 .{ }^{6}$ Antidepressants are widely used in the treatment of MDD, and a host of new generation antidepressants have been approved into the market in the past two to three decades. Given the enormity of burdens due to MDD on patients and society, various institutions worldwide have produced CPGs for physicians. It was as early as in 1993 that American Psychiatric Association (APA), ${ }^{7}$ British Association for Psychopharmacology (BAP) ${ }^{8}$ and Agency for Health Care Policy and Research (AHCPR) ${ }^{9}$ proposed the first version of their guidelines, followed by other associations like National Institute for Health and Care Excellence (NICE) from $\mathrm{UK}^{10}$ and Canadian Network for Mood and Anxiety Treatments from Canada. ${ }^{11}$ Most of these guidelines have been updated every several years since.

However, there are still several important barriers that may impede physicians and patients from making optimal clinical decisions. First, it has become increasingly known that a substantive amount of trials of antidepressants remain unpublished and were not included in contemporary SRs, a fact which most likely introduces publication bias into the results of SRs and thus undermines the credibility of guidelines. ${ }^{12}$ Second, it has been reported that there is approximately 8 months to more than 1-year lag between the last search date in the literature and the publication of an SR, ${ }^{1314}$ and in total 2.5-6.5 years interval between the publication dates of the latest primary studies and the publication date of SRs. ${ }^{15}$ It was estimated that $7 \%$ of the published SRs were already out of date at the time of publication. ${ }^{16}$ Third, few of the available CPGs provide specific and precise recommendations in the choice of antidepressants, as pooled evidence has been given conflicting interpretations. ${ }^{17} 18$ Fourth, physicians' behaviours are notoriously difficult to influence. ${ }^{19-21}$ The road from randomised evidence to actual practices thus appears formidable. ${ }^{22}$

The aim of the present study is to examine how the accumulating body of evidence should have been assembled and reflected in guidelines, and then transferred into real practices in the prescription of new generation antidepressants for MDD through the past three decades. The evidence we will consider comes from a uniquely comprehensive dataset prepared by Group of Researchers Investigating Specific Efficacy of individual Drugs for Acute depression (GRISELDA). ${ }^{23}{ }^{24}$ It comprises data from 522 published and unpublished double-blind randomised controlled trials (RCTs) of new generation and other antidepressants in the acute treatment of depression. Capitalising on this, we will use cumulative network MA (cNMA) to summarise the development of accumulated evidence over time. ${ }^{25-27}$ When two or more treatment alternatives are available, as in the case of MDD, NMA can summarise the relative effects of all treatments options by combining both direct and indirect comparisons ${ }^{28}$; because they use all direct and indirect evidence, they can produce strong evidence concerning relative efficacy more often and earlier than conventional, pairwise MAs. ${ }^{29}$ We will examine the specific recommendations in the internationally representative CPGs. The real-world prescription practices will be collated from Medical Expenditure Panel Survey (MEPS) in USA, which is a large-scale yearly healthcare survey of representative samples of families and individuals in the USA. ${ }^{30}$ We predict that there are important gaps in the evidence synthesis and implementation process. Our study will evaluate and narratively summarise how big these gaps are and where mainly they exist, and will provide valuable information necessary to direct future paths to bridge the gaps from scientific evidence to real-world clinical practice.

\section{METHODS}

SRs of RCTs of new generation antidepressants and CNMA Study eligibility

The cNMAs in this study will be based on the SR which has identified all published and unpublished double-blinded head-to-head RCTs comparing any of the following new generation antidepressants in the acute phase treatment of adults with MDD up to January $2016^{2324}$ : we will consider all new generation antidepressants approved by drug agencies of USA, Europe or Japan including agomelatine, bupropion, citalopram, desvenlafaxine, duloxetine, escitalopram, fluoxetine, fluvoxamine, levomilnacipran, milnacipran, mirtazapine, paroxetine, reboxetine, sertraline, venlafaxine, vilazodone and vortioxetine.

The GRISELDA dataset also contains two older tricyclic antidepressants (TCAs) recommended by WHO Model List of Essential Medicines (amitriptyline and clomipramine) and two drugs with distinct effect and side effect profiles (trazodone and nefazodone): they will constitute evidence set ${ }^{31}$ in the NMAs which serves to connect and strengthen the network.

We will include in the MA only study arms which administered the drugs within dose ranges approved by the regulatory agencies. Because the inclusion of placebo-controlled trials may violate the transitivity assumption of NMA in depression studies, ${ }^{32-36}$ and because we will be comparing the evidence from cNMA with real-world practice, we will only include RCTs comparing active drugs and exclude placebo-controlled studies in the current cNMAs. 
Study identification and data extraction

GRISELDA has searched the following databases without language restrictions for published articles: Cochrane Central Register of Controlled Trials, CINAHL, EMBASE, LiLACS, MEDLINE, MEDLINE In-Process and PsycINFO up to January 2016. We performed manual searches of references of included articles. We also searched for published, unpublished and ongoing RCTs in drug-approval agencies' documents, company websites, international trial registries and through contact with pharmaceutical companies and other relevant organisations. Where necessary, we contacted study authors directly to get online supplementary materials for further information. The risk of bias of each included study was assessed according to the guide proposed in the Cochrane Collaboration Handbook. ${ }^{37}$

Study identification, data extraction and risk of bias assessment of the included studies were all performed independently by two researchers, and any disagreements were resolved through discussion or in consultation with a third reviewer. For further details of the SR process, please see the published protocol and the main GRISELDA paper. ${ }^{23} 24$

\section{Statistical analyses}

We will conduct cNMA every 5 years in consideration of the speed of accumulating new trials and publication of guidelines for MDD. According to previous studies which indicated that the latest search of database was about 1 year before publication of SRs, ${ }^{13-15}$ cNMA at any time point will include all trials completed up to 1 year before that date. For trials whose completion year is not available, publication date will be used; when neither is known, the date of approval of the drug by regulatory agencies will be used as the study completion date. Thus, studies with a known completion year before the proposed cNMA will be included in the relevant cNMA even if the data were published after that year. Only such cNMA can present the best available, publication bias-free evidence, on which practice guidelines should ideally be founded.

For NMA, transitivity assumption is the principle. Since we have confirmed the transitivity assumption of the whole dataset in the final NMA, ${ }^{24}$ we will not validate it at every time point reanalysis. In our previous study, we investigated the distribution of the following potential effect modifiers across treatment comparison: (1) study year; (2) sponsorship; (3) depressive severity at baseline; (4) dosing schedule; (5) proportion of participants allocated to placebo; (6) number of recruiting centres (singlecentre vs multicentric studies). We evaluated consistency and heterogeneity in the entire network using various tests and metrics and we have found little evidence of inconsistency and heterogeneity. ${ }^{24}$

Our primary outcomes are (1) efficacy of the intervention in terms of response rate defined as the proportion of patients who showed a reduction of at least $50 \%$ on the total depression severity score compared with baseline and (2) acceptability of the intervention in terms of all-cause discontinuation rate defined as the proportion of patients who leave the study early for any reason. We have extracted the outcome data at a time point as close to 8 weeks as possible: if outcomes at 8 weeks were not available, we recorded data between 4 and 12 weeks.

We will not adjust for multiple comparisons in successive NMAs as we are not testing particular hypotheses for specific comparisons among antidepressants.

We will use the surface under the cumulative ranking curve to estimate ranking probabilities for each intervention with regard to the two primary outcomes. We will show ranked forest plots of ORs with 95\% CI in comparison with a common comparator as a sensitivity analysis: we will use fluoxetine as the common comparator as it is the most frequently used drug in the trials network. ${ }^{24}$

We will use STATA V.14 (StataCorp) to conduct cNMA. $^{38} 39$

\section{Identification and extraction of recommendations in guidelines}

We will use a series of CPGs as our benchmark in comparisons. English written guidelines proposed by government agencies (such as NICE) or professional academic societies (such as APA) will be included. Due to the ambiguity and subjectivity associated with the Appraisal of Guidelines Research and Evaluation II (AGREE) instrument for quality assessment of guidelines, ${ }^{40}$ we will not apply AGREE instrument in the appraisal of guidelines, but we will describe the methodology of each guideline development in online supplementary materials. As a result, we will extract recommendations for specific antidepressants or for specific classes of antidepressants from internationally representative guidelines listed below:

1. Practice guidelines for the treatment of patients with MDD published by APA, in 1993, 2000, 2010, respectively. $^{74142}$

2. Guidelines for management of depression proposed by NICE, in 2004, 2009 and 2018. ${ }^{10} 4344$

3. Guidelines for treating depressive disorders with antidepressants proposed by BAP, in 1993, 2000, 2008 and $2015 .^{845-47}$

4. CPGs for treatment of major depression proposed by AHCPR in $1993 .^{9}$

We will extract recommendations with respect to acute phase treatment for adult patients with a primary diagnosis of unipolar non-psychotic major depression. We define recommendations as statements used in CPGs involving the words like 'recommend', 'must', 'necessary', 'should', 'appropriate' or other words indicating instructions from guidelines. Basic information of the guideline (institution, year, targeted patients, diagnostic criteria, etc), pharmacotherapy recommendations as acute phase treatment (particular drugs and categories recommended and least recommended, severity of the disease, etc), pharmacotherapy recommendations for patients who have no satisfactory response to initial treatment will be recorded in detail, in order to be compared with results from cNMA and real world. We will also collect the quality of evidence 
and strength of recommendations backing extracted recommendations. Two researchers will independently identify recommendations and extract information from CPGs. Any disagreement will be resolved through discussion or in consultation with a third researcher.

\section{Real-world prescriptions data extraction}

We will extract real-world prescription data from the MEPS database.$^{30}$ MEPS is a database composed of largescale surveys of families and individuals and their medical providers, collecting data on the use of specific health services, the cost and the health insurance in the USA. The survey started in 1996. MEPS has two major components, the household component and the insurance component, both of which provide data on a yearly basis. In the household component, a representative sample of families and individuals are interviewed every year and detailed information including demographic characteristics, health status, use of medical services, specific medications, cost for each person is collected. Each annual MEPS household sample size is about 15000 households. This set of households is a subsample of households participating in the previous year's National Health Interview Survey (NHIS) led by the National Center for Health Statistics. The NHIS sampling frame gives a nationally representative sample of the non-institutionalised population in the USA, and oversamples of blacks and Hispanics. In 2006, after implementing a new sample design, Asian population has been included in order to deal with the oversampling issues. To correct for oversampling and for non-response, each participant is given a weight adjusting for non-response over time and some poststratification variables (region, race/ethnicity, sex, age, poverty status and etc), in order to produce national estimates. ${ }^{30}$ MEPS database has been used for the analysis of health expenditures and real-world prescriptions of specific drugs in several studies. ${ }^{48-50}$

\section{Participants}

We will include those who have been classified as 'depressive disorders' in the category of ' 657 mood disorder', of either sex and aged 18 years or older. The corresponding International Statistical Classification of Diseases and Related Health Problems 10th Revision (ICD-10) numbers are: 29620, 29621, 29622, 29623, 29624, 29625, 29626, 29630, 29631, 29632, 29633, 29634, 29635, 29636, 311. MEPS database only code the diagnosis for the first three digits using ICD numbers $(296,311)$.

\section{Interventions}

Of the 21 antidepressants included in the GRISELDA network, the following drugs will not be included among the drugs prescribed for depressive disorders in MEPS: agomelatine and reboxetine have not been approved by US Food and Drug Administration (FDA) for marketing in USA, fluvoxamine has an indication for obsessive-compulsive disorder but not for depression and milnacipran for fibromyalgia only. On the other hand,
Table 1 Time periods for comparisons in our study

\begin{tabular}{llll}
\hline $\begin{array}{l}\text { Trials } \\
\text { (completion } \\
\text { year*) }\end{array}$ & $\begin{array}{l}\text { Cumulative } \\
\text { NMAs }\end{array}$ & $\begin{array}{l}\text { CPGs } \\
\text { (publication } \\
\text { year) }\end{array}$ & $\begin{array}{l}\text { Real-world } \\
\text { prescriptions } \\
\text { (MEPS data } \\
\text { file year) }\end{array}$ \\
\hline Present & Present & 2016 to present & - \\
\hline 2009 & 2010 & 2010 to 2015 & 2015 \\
\hline 2004 & 2005 & 2005 to 2009 & 2010 \\
\hline 1999 & 2000 & 2000 to 2004 & 2005 \\
\hline 1994 & 1995 & 1995 to 1999 & 2000 \\
\hline 1989 & 1990 & 1990 to 1994 & $1996 \dagger$ \\
\hline
\end{tabular}

*For trials whose completion year is not available, publication date will be used; when neither is known, the date of approval of the drug by regulatory agencies will be used as its completion date. †As MEPS database started with 1996, so we will use data from 1996 for the analysis of prescriptions.

CPGs, clinical practice guidelines; MEPS, Medical Expenditure Panel Survey; NMAs, network meta-analyses.

there are many antidepressants, other than new generation antidepressants, which may have been prescribed especially in older days, such as imipramine, desipramine, doxepin or nortriptyline. Although our focus of comparison is new generation antidepressants, we will extract the frequency of prescription of all antidepressants in the MEPS study. Using the National Drug Code Directory, ${ }^{51}$ which included all registered drugs in FDA, we identified all antidepressants ever used in the USA as the following: (1) TCAs (Established Pharmacologic Class [EPC]): amitriptyline, amoxapine, clomipramine, desipramine, doxepin, imipramine, nortriptyline, protriptyline, trimipramine; (2) Serotonin reuptake inhibitor (EPC): citalopram, escitalopram, fluoxetine, nefazodone, paroxetine, sertraline, trazodone; (3) Serotonin and norepinephrine reuptake inhibitor (EPC): desvenlafaxine, duloxetine, venlafaxine, levomilnacipran; (4) No pharm class: bupropion, mirtazapine, vilazodone, vortioxetine. All the 24 listed antidepressants will be searched in MEPS during data extraction.

\section{Data extraction}

We will extract prescription data for antidepressants listed above in the year 1996, 2000, 2005, 2010 and 2015 (table 1). Since diagnostic labels available MEPS data cannot distinguish unipolar depression from bipolar depression and psychotic depression, both of which require different drug treatments than unipolar depression, we will exclude those patients who are concomitantly taking mood stabilisers or any antipsychotics. According to the National Drug Code Directory, ${ }^{51}$ mood stabiliser (EPC) include: carbamazepine, divalproex, lamotrigine, lithium, valproate, valproic acid; and phenothiazine (EPC), typical antipsychotics (EPC) and atypical antipsychotics (EPC) include: aripiprazole, asenapine, brexpiprazole, cariprazine, chlorpromazine, clozapine, fluphenazine, haloperido, iloperidone, loxapine, lurasidone, molindone, olanzapine, paliperidone, 


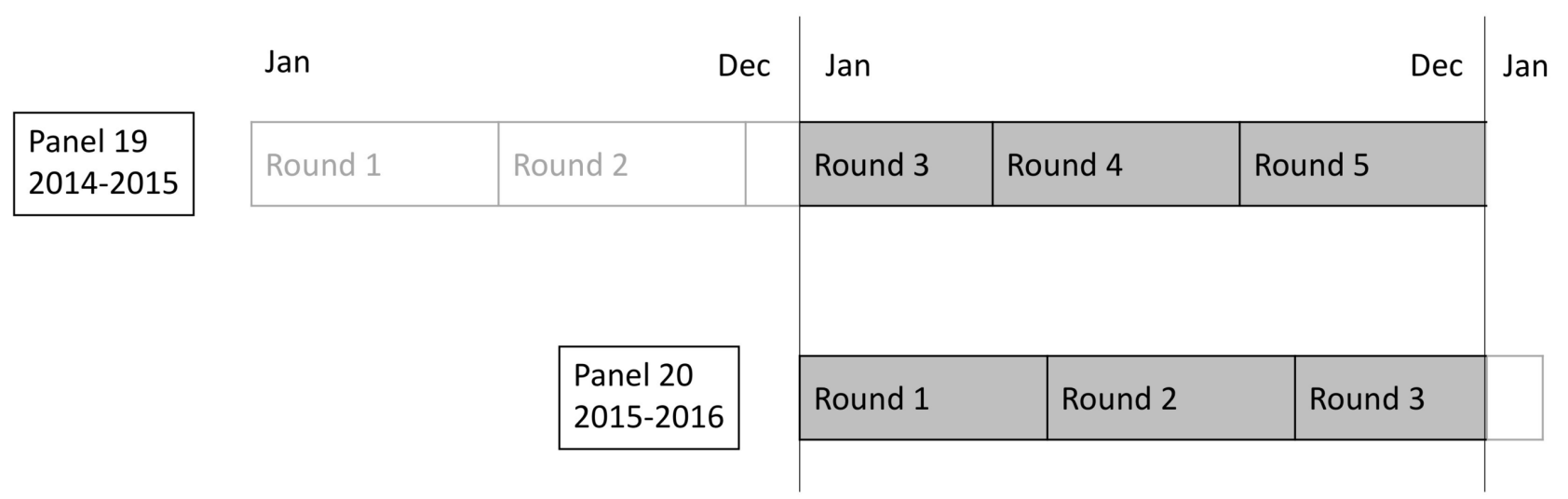

Figure 1 Survey structure of 2015 file in Medical Expenditure Panel Survey (MEPS). ${ }^{30}$

perphenazine, pimavanserin, quetiapine, risperidone, thioridazine, thiothixene, ziprasidone. We will exclude all participants taking any of these drugs concomitantly with an antidepressant.

Every participant in one MEPS cohort (called 'panel' in MEPS) is interviewed five times (called 'rounds') for 2 years. For example, the file of 2015 contains the 2015 portion of round 3 and rounds 4 and 5 for panel 19, as well as rounds 1, 2 and the 2015 portion of round three for panel 20 (see figure 1 ) ${ }^{30}$ We will check the prescription of antidepressants at round 2 from panel 20 and round 4 from panel 19 (similar patterns in other given years), because each participant, either from panel 19 or 20, must have this intermediate round of interview within 2015, and we will use it as a cross-sectional investigation. We will use the total number of patients with the diagnosis of depression and who are on antidepressant monotherapy but not taking mood stabilisers and antipsychotics (listed above) concomitantly at this round as denominator, and the prescription for a particular drug as monotherapy as numerator. Given lack of precise information for medication schedule (eg, start time of diagnosis, primary medication, time of start and change of medication), we will not be able to restrict the prescriptions to inception prescriptions in the acute phase treatment of MDD, and the results will include both incident and prevalent uses. As MEPS dataset gives each participant a sample weight according to non-response and poststratification variables, we will use the sample weights to estimate the nationally representative number of patients on a specific therapy. Finally, the ranking of the of prescriptions will then be based on the estimated numbers of prescriptions in the general US population.

We will use Python V.3.6 to extract data from MEPS dataset.

\section{Comparison between cNMA, CPGs and real-world prescriptions}

We propose that a cNMA at a specific time point (eg, 2000) should be taken as a reference for any guidelines that are going to be published in the next 5 years (eg, 2000-2004), and then inform real-world prescriptions afterwards (eg,
2005 and after). We will therefore compare the results of NMA, CPG recommendations and real-world practice in these corresponding years, and the trend between these time points will also be taken into considerations. Table 1 shows the time periods for comparisons. We will assess if the recommendable sets estimated from cNMA (ie, drugs which have good balance between efficacy and acceptability) are reflected in the drugs recommended by guidelines, which then will be compared with the rankings in actual clinical practice. Moreover, as MEPS is a database from the USA, we will attempt to compare results from MEPS with the US guidelines APA. We will describe the possible differences and explore the time lag from the ideally available evidence to guideline recommendations and from guideline recommendations to the real-world practices. We will also investigate the potential factors which may influence the actual prescriptions including side effects profiles of specific drugs, year of FDA approval, the price of the drug while on patent, and the year of patent expiration of each drug.

\section{Patient and public involvement}

This research protocol was written without patient involvement. Patients were not invited to comment on the study design and were not consulted to develop patient-relevant outcomes or interpret the results. Patients were not expected to contribute to conducting this study and to the writing of this document for readability or accuracy.

\section{DISCUSSION}

We have presented the study protocol for investigating the gaps between ideally synthesised evidence, guideline recommendations and real-world clinical practices in the prescriptions of new generation antidepressants for unipolar MDDs.

Our study has several strengths. It is one of the first studies to make comparisons between pooled evidence derived from cNMAs, recommendations from guidelines and real-world clinical practices. In the cNMA, we will use the comprehensive dataset from our previous study, ${ }^{24}$ 
which included both published and unpublished trials. And we will use MEPS database, which collect detailed information of health status and specific medications from a representative sample in the USA, as a reflection of real-world prescription profile. We expect this study to show the gaps between evidence and practice, and our findings will inform future developments of EBM. For instance, the cNMA used in this study may eventually evolve into prospectively designed sequential NMA which can be used to continuously update evidence and contribute to living guidelines, which is a guideline that is updated as soon as new evidence becomes available, so that it can provide timely and trustworthy suggestions for decision-makers. ${ }^{15253}$

There are some limitations to our study. First, MEPS does not allow us to extract very precise information, including whether the antidepressant is being used as first-line or later treatments. This may lower the comparability between the cNMA of acute phase treatment studies of unipolar depression and the real-world practices, as patients on continuation/maintenance treatment may continue using the same antidepressants after guideline recommendations for acute phase treatment change. However, it must be noted that three-quarters of patients who initiate antidepressant treatment discontinue the drug within 90 days $^{54}$, suggesting that the majority of the patients in MEPS database represent initial prescriptions. Second, MEPS is a US database so we will not be able to make comparisons between guideline recommendations and actual practices in other countries. Lastly, the reasons behind the differences between evidence and practice may be very complicated and we will need to factor in various potential confounding factors such as the side effects, marketing efforts of pharmaceutical companies and local regulations such as price or patent status of a particular drug. ${ }^{15556}$

In conclusion, our study represents a unique inquiry into gaps from evidence to real-world practices, and may provide valuable insights into future developments of EBM.

\section{Author affiliations}

${ }^{1}$ Department of Health Promotion and Human Behavior, School of Public Health in the Graduate School of Medicine, Kyoto University, Kyoto, Japan

${ }^{2}$ School of Medicine, Paris Descartes University, Paris, France

${ }^{3}$ Epidemiology and Statistics, Sorbonne Paris Cité Research Center, METHODS

Team, Paris, France

${ }^{4}$ Cochrane France, Paris, France

${ }^{5}$ Hospital Care Research Unit, Hyogo Prefectural Amagasaki General Medical Center, Amagasaki, Japan

${ }^{6}$ Department of Health Sciences, Università degli Studi di Milano, Milan, UK ${ }^{7}$ Department of Psychiatry, University of Oxford, Oxford, UK

${ }^{8}$ Institute of Social and Preventive Medicine, University of Bern, Bern, Switzerland

Acknowledgements We would like to thank all the constructive comments and advices from the members from the meta-epidemiological study group in the School of Public Health, Kyoto University.

Contributors YL and TAF conceived the study and drafted the protocol. ACh, YK, $\mathrm{EGO}, \mathrm{YO}, \mathrm{ACi}$ and GS assisted in the study design and critically revised the protocol. All authors gave final approval of the version to be published.
Funding This study was supported in part by JSPS Grant-in-Aid for Scientific Research (Grant Number17k19808) to TAF.

Disclaimer The funder has no role in study design, data collection, data analysis, data interpretation, writing of the report or in the decision to submit for publication.

Competing interests TAF has received lecture fees from Janssen, Meiji, Mitsubishi-Tanabe, MSD and Pfizer. He has received research support from Mitsubishi-Tanabe and Mochida. ACi is supported by the NIHR Oxford Cognitive Health Clinical Research Facility, and was expert witness for Accord Healthcare for a patent issue about quetiapine extended release.

Patient consent Not required.

Ethics approval This study does not require institutional review board approval.

Provenance and peer review Not commissioned; externally peer reviewed.

Open access This is an open access article distributed in accordance with the Creative Commons Attribution Non Commercial (CC BY-NC 4.0) license, which permits others to distribute, remix, adapt, build upon this work non-commercially, and license their derivative works on different terms, provided the original work is properly cited, appropriate credit is given, any changes made indicated, and the use is non-commercial. See: http://creativecommons.org/licenses/by-nc/4.0/.

\section{REFERENCES}

1. Djulbegovic B, Guyatt GH. Progress in evidence-based medicine: a quarter century on. Lancet 2017;390:415-23.

2. Haynes RB. Of studies, syntheses, synopses, summaries, and systems: the " $5 \mathrm{~S}$ " evolution of information services for evidencebased healthcare decisions. Evid Based Med 2006;11:162-4.

3. Murray CJ, Barber RM, Foreman KJ, et al. Global, regional, and national disability-adjusted life years (DALYs) for 306 diseases and injuries and healthy life expectancy (HALE) for 188 countries, 1990-2013: quantifying the epidemiological transition. Lancet 2015;386:2145-91.

4. GBD 2015 DALYs and HALE Collaborators. Global, regional, and national disability-adjusted life-years (DALYs) for 315 diseases and injuries and healthy life expectancy (HALE), 1990-2015: a systematic analysis for the Global Burden of Disease Study 2015. Lancet 2016;388:1603-58

5. WHO. Depression: fact sheet. Geneva: World Health Organisation, 2017.

6. Ferrari AJ, Charlson FJ, Norman RE, et al. Burden of depressive disorders by country, sex, age, and year: findings from the global burden of disease study 2010. PLoS Med 2013;10:e1001547.

7. American Psychiatric Association. Practice guideline for major depressive disorder in adults. Am J Psychiatry 1993;150:1-26.

8. Montgomery SA, Bebbington P, Cowen P, et al. Guidelines for treating depressive illness with antidepressants: a statement from the british association for psychopharmacology. J Psychopharmacol 1993;7(1 Suppl):19-23.

9. AHCPR Clinical Practice Guidelines N. Depression in Primary Care (Volume 2: Treatment of Major Depression, 1993. https://www.ncbi. nlm.nih.gov/books/NBK52234/\#!po=16.6667.

10. Health NCCfM. Management of depression in primary and secondary care, 2004. https://www.nice.org.uk/guidance/CG23.

11. Kennedy SH, Lam RW, Parikh SV, et al. Canadian Network for Mood and Anxiety Treatments (CANMAT) clinical guidelines for the management of major depressive disorder in adults. Introduction. $J$ Affect Disord 2009;117:S1-S2.

12. Turner EH, Matthews AM, Linardatos E, et al. Selective publication of antidepressant trials and its influence on apparent efficacy. $N$ Engl $J$ Med 2008;358:252-60.

13. Beller EM, Chen JK, Wang UL, et al. Are systematic reviews up-todate at the time of publication? Syst Rev 2013;2:36.

14. Sampson M, Shojania KG, Garritty C, et al. Systematic reviews can be produced and published faster. J Clin Epidemiol 2008;61:531-6.

15. Elliott JH, Turner T, Clavisi O, et al. Living systematic reviews: an emerging opportunity to narrow the evidence-practice gap. PLoS Med 2014;11:e1001603.

16. Shojania KG, Sampson M, Ansari MT, et al. How quickly do systematic reviews go out of date? A survival analysis. Ann Intern Med 2007;147:224-33.

17. Cipriani A, Furukawa TA, Salanti G, et al. Comparative efficacy and acceptability of 12 new-generation antidepressants: a multipletreatments meta-analysis. Lancet 2009;373:746-58.

18. Gartlehner G, Hansen RA, Morgan LC, et al. Comparative benefits and harms of second-generation antidepressants for treating major 
depressive disorder: an updated meta-analysis. Ann Intern Med 2011:155:772-85.

19. Forsetlund $L$, Bjorndal $A$, Rashidian $A$, et al. Continuing education meetings and workshops: effects on professional practice and health care outcomes. Cochrane Database Syst Rev 2009;2:CD003030.

20. Ivers N, Jamtvedt G, Flottorp S, et al. Audit and feedback: effects on professional practice and healthcare outcomes. Cochrane Database Syst Rev 2012;6:CD000259.

21. O'Brien MA, Rogers S, Jamtvedt G, et al. Educational outreach visits: effects on professional practice and health care outcomes. Cochrane Database Syst Rev 2007;4:CD000409.

22. Balas EA, Boren SA. Managing clinical knowledge for health care improvement. Yearb Med Inform 2000;1:65-70.

23. Furukawa TA, Salanti G, Atkinson LZ, et al. Comparative efficacy and acceptability of first-generation and second-generation antidepressants in the acute treatment of major depression: protocol for a network meta-analysis. BMJ Open 2016;6:e010919.

24. Cipriani A, Furukawa TA, Salanti G, et al. Comparative efficacy and acceptability of 21 antidepressant drugs for the acute treatment of adults with major depressive disorder: a systematic review and network meta-analysis. Lancet 2018;391:1357-66.

25. Antman EM, Lau J, Kupelnick B, et al. A comparison of results of meta-analyses of randomized control trials and recommendations of clinical experts. Treatments for myocardial infarction. JAMA 1992;268:240-8.

26. Lau J, Antman EM, Jimenez-Silva J, et al. Cumulative metaanalysis of therapeutic trials for myocardial infarction. N Engl J Med 1992;327:248-54.

27. Rouse B, Cipriani A, Shi Q, et al. Network meta-analysis for clinical practice guidelines: a case study on first-line medical therapies for primary open-angle glaucoma. Ann Intern Med 2016;164:674-82.

28. Cipriani A, Higgins JP, Geddes JR, et al. Conceptual and technical challenges in network meta-analysis. Ann Intern Med 2013;159:130-7.

29. Nikolakopoulou A, Mavridis D, Furukawa TA, et al. Living network meta-analysis compared with pairwise meta-analysis in comparative effectiveness research: empirical study. BMJ 2018;360:k585.

30. Quality USAfHRa. Medical Expenditure Panel Survey (MEPS). https:// meps.ahrq.gov/mepsweb/.

31. Ades AE, Caldwell DM, Reken S, et al. Evidence Synthesis of Treatment Efficacy in Decision Making: A Reviewer's Checklist. London, 2012.

32. Rutherford BR, Sneed JR, Roose SP. Does study design influence outcome?. The effects of placebo control and treatment duration in antidepressant trials. Psychother Psychosom 2009;78:172-81.

33. Sinyor M, Levitt AJ, Cheung AH, et al. Does inclusion of a placebo arm influence response to active antidepressant treatment in randomized controlled trials? Results from pooled and metaanalyses. J Clin Psychiatry 2010;71:270-9.

34. Furukawa TA, Cipriani A, Atkinson LZ, et al. Placebo response rates in antidepressant trials: a systematic review of published and unpublished double-blind randomised controlled studies. Lancet Psychiatry 2016;3:1059-66.

35. Furukawa TA, Cipriani A, Leucht S, et al. Is placebo response in antidepressant trials rising or not? A reanalysis of datasets to conclude this long-lasting controversy. Evid Based Ment Health. In Press. 2018;21:1-3.

36. Salanti G, Chaimani A, Furukawa TA, et al. Impact of placebo arms on outcomes in antidepressant trials: systematic review and metaregression analysis. Int J Epidemiol. In Press. 2018;47:1454-64.
37. Collaboration TC. Cochrane Handbook for Systematic Reviews of Interventions Version 5.1.0. 2011. http://www.cochrane-handbook. org.

38. Salanti ACG. Visualizing assumptions and results in network metaanalysis: The network graphs package. Stata Journal, StataCorp LP 2015;15:905-50.

39. White IR. Network meta-analysis. Stata Journal, StataCorp LP 2015;15:951-85.

40. Scott NA, Moga C, Harstall C. Making the AGREE tool more userfriendly: the feasibility of a user guide based on Boolean operators. $J$ Eval Clin Pract 2009;15:1061-73.

41. American Psychiatric Association. Practice guideline for the treatment of patients with major depressive disorder (revision). Am J Psychiatry 2000;157:1-45.

42. Association AP. Practice Guideline for the Treatment of Patients With Major Depressive Disorder. 3rd Edition, 2010. Available from. http://www.psychiatryonline.com/pracGuide/pracGuideTopic 7. aspx.

43. NICE. Depression in adults: recognition and management, 2010. https://www.nice.org.uk/guidance/cg90.

44. NICE. Depression in adults: treatment and management, 2018.

45. Anderson IM, Nutt DJ, Deakin JF. Evidence-based guidelines for treating depressive disorders with antidepressants: a revision of the 1993 British Association for Psychopharmacology guidelines. British Association for Psychopharmacology. J Psychopharmacol 2000;14:3-20.

46. Anderson IM, Ferrier IN, Baldwin RC, et al. Evidence-based guidelines for treating depressive disorders with antidepressants: a revision of the 2000 British Association for Psychopharmacology guidelines. J Psychopharmacol 2008;22:343-96.

47. Cleare A, Pariante CM, Young AH, et al. Evidence-based guidelines for treating depressive disorders with antidepressants: A revision of the 2008 British Association for Psychopharmacology guidelines. $J$ Psychopharmacol 2015;29:459-525.

48. Lichtenberg FR. Are the benefits of newer drugs worth their cost? Evidence from the 1996 MEPS. Health Aff 2001;20:241-51.

49. Miller JD, Foster T, Boulanger L, et al. Direct costs of COPD in the U.S.: an analysis of Medical Expenditure Panel Survey (MEPS) data. COPD 2005;2:311-8.

50. Sullivan PW, Ghushchyan VH, Slejko JF, et al. The burden of adult asthma in the United States: evidence from the Medical Expenditure Panel Survey. J Allergy Clin Immunol 2011;127:363-9.

51. FDA. National Drug Code Directory, 2018. https://www.fda.gov/ drugs/informationondrugs/ucm142438.htm.

52. Akl EA, Meerpohl JJ, Elliott J, et al. Living systematic reviews: 4. Living guideline recommendations. J Clin Epidemiol 2017;91:47-53.

53. Elliott JH, Synnot A, Turner T, et al. Living systematic review: 1 . Introduction-the why, what, when, and how. J Clin Epidemiol 2017:91:23-30.

54. Olfson M, Marcus SC, Tedeschi M, et al. Continuity of antidepressant treatment for adults with depression in the United States. Am J Psychiatry 2006;163:101-8.

55. Clemens J, Gottlieb JD. Do physicians' financial incentives affect medical treatment and patient health? Am Econ Rev 2014;104:1320-49.

56. Yeh JS, Franklin JM, Avorn J, et al. Association of industry payments to physicians with the prescribing of brand-name statins in massachusetts. JAMA Intern Med 2016;176:763-8. 